\title{
Comparative Analysis of Automatic Vehicle Classification Techniques: A Survey
}

\author{
Kanwal Yousaf, Arta Iftikhar, Ali Javed \\ Department of Software Engineering, University of Engineering and Technology \\ Taxila, Pakistan \\ E-mail: kanwal_400@yahoo.com, artaiftikhar@yahoo.com, ali.javed@uettaxila.edu.pk
}

\begin{abstract}
Vehicle classification has emerged as a significant field of study because of its importance in variety of applications like surveillance, security system, traffic congestion avoidance and accidents prevention etc. So far numerous algorithms have been implemented for classifying vehicle. Each algorithm follows different procedures for detecting vehicles from videos. By evaluating some of the commonly used techniques we highlighted most beneficial methodology for classifying vehicles. In this paper we pointed out the working of several video based vehicle classification algorithms and compare these algorithms on the basis of different performance metrics such as classifiers, classification methodology or principles and vehicle detection ratio etc. After comparing these parameters we concluded that Hybrid Dynamic Bayesian Network (HDBN) Classification algorithm is far better than the other algorithms due to its nature of estimating the simplest features of vehicles from different videos. HDBN detects vehicles by following important stages of feature extraction, selection and classification. It extracts the rear view information of vehicles rather than other information such as distance between the wheels and height of wheel etc.
\end{abstract}

Index Terms - Vehicle classification, Video based vehicle classification, Vehicle classification algorithms

\section{INTRODUCTION}

No doubt the rapid rise in social economy has severe impact on different areas of life including transportation system. Increase in vehicles lead to some serious issues such as vehicle congestions, accidents and car robberies etc. In order to cope up with these issues ITS (Intelligent Transportation System) turn its focus on vehicle classification and detection [1]. In recent years vehicle classification has been renowned as a broader field of study in vehicle recognition. Vehicle classification is an efficient technique used for transportation systems such as toll plaza, traffic surveillance, traffic congestion avoidance, terrorist activities inspection etc. Different toll stations defined a standard for classifying vehicles. This standard is based on different types of vehicles. There are basically three main types of vehicles as shown in Table I.
TABLE I MAJOR TYPE OF VEHICLE CATEGORIES

\begin{tabular}{|c|c|c|}
\hline $\begin{array}{c}\text { Vehicles } \\
\text { Type }\end{array}$ & $\begin{array}{c}\text { Assigned } \\
\text { Code }\end{array}$ & \multicolumn{1}{c|}{ Vehicle Description } \\
\hline $\begin{array}{c}\text { Light Weight } \\
\text { Vehicles }\end{array}$ & Category-A & $\begin{array}{l}\text { Small Cars or Vehicles with the } \\
\text { load no more than 1 ton or bus } \\
\text { with sets no more than 10 }\end{array}$ \\
\hline $\begin{array}{c}\text { Intermediate } \\
\text { Vehicles }\end{array}$ & Category-B & $\begin{array}{l}\text { Vans/Suzuki etc or Vehicles } \\
\text { with the load larger than 1 ton } \\
\text { but less than 7 tons or the bus } \\
\text { with set larger than 10 and not } \\
\text { more than 28 }\end{array}$ \\
\hline $\begin{array}{c}\text { Heavy } \\
\text { Vehicles }\end{array}$ & Category-C & $\begin{array}{l}\text { Truck/buses or Vehicles with the } \\
\text { load greater than 7 tons or the } \\
\text { bus with set larger than 28 }\end{array}$ \\
\hline
\end{tabular}

Two common techniques are used for the classification of vehicles as mentioned in Fig.1.

1) Hardware Based Vehicle Classification

2) Software Based Vehicle Classification

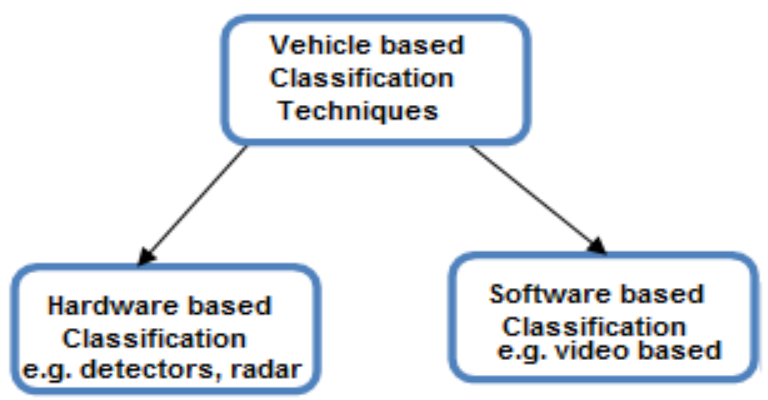

Figure.1 Techniques for Vehicle-based Classification Mechanism

Hardware based vehicle classification include different tools such as magnetic, radar, infrared detectors etc. This classification technique has various drawbacks:

- Such detectors are large in size and have difficult maintenance.

- Installation cost is very high

- Its installation causes damage to the road.

- Costly detectors

- Limited information 
While software based vehicle classification includes video based detection. Video based detection has many advantages over hardware based classification such as

- Easy Installation

- No damage to roads

- Very less installation and maintenance cost

- Provide much more information than hardware based detectors, can be adjusted easily to provide wider view and more information about traffic.

- Friendly to environment and reduction of pollution

Though video based classifications have few limitations such as slow processing, low real time capacity but still are far better than hardware based classifications. Various classification algorithms have been proposed based on infrared, ultrasonic, laser and video detection based technology. Because of easy installation, maintenance, multilane detection and availability of synchronous information about vehicles made video based detection more advantageous than other technologies.

The rest of the paper is organized as follows. In Section II, we explained the importance of using video based vehicle classification over the hardware based classification techniques. Most recent and commonly implemented video based classification algorithms are mentioned in Section III. We evaluated and compare these algorithms in Section IV. The comparison table is also maintained to get an easy understanding of all these analyzed algorithms.

\section{LITERATURE REVIEW}

Avery, Wang and Rutherford [2] proposed digital image processing algorithm for length based vehicle classification. They used streams of images captured from un-calibrated video camera. They actually compare the length of different vehicles in order to estimate the truck volumes and eliminated the needs of different complex calibration systems. They implemented length classification algorithm on trucks and got $92 \%$ accuracy under certain conditions.

Zhang, Avery and Wang [3] described that length based classification technique is important for transportation planning but this data cannot be measurable by single hardware detector. So they developed Video-Based Vehicle Detection and Classification System (VVDC) for the collection of truck related data. They divided VVDC system into six modules such as capturing of live video, get user input, extraction of background, vehicle detection, shadow removal and length based classification. Their defined algorithm got $97 \%$ accuracy for classifying trucks. But this VVDC system cannot handle the longitudinal vehicle occlusions, server camera movements and head-light reflection problems.
$\mathrm{Wu}$, Zang, Xu, and Song [4] reviewed different video vehicle classification techniques and suggested that video based vehicle classification is more advantageous than any other classification technique. They explained different video based vehicle classification techniques such as optical flow field, background removal, edge detection, frame differential and gray comparison techniques. They highlighted that no doubt video detectors are advantageous but still have many unresolved issues. These issues can be resolved by using better and appropriate vehicle classification algorithm.

Wang [5] presented an approach for detecting the moving vehicles and removal of cast shadows for video based traffic monitoring and control system. He developed an algorithm to handle non-stationary background for vehicle detection in live video streams. This method significantly improved the overall performance of vehicle detection and shadow removal process.

Similarly Qin [6] used the basic technique for vehicle classification by analyzing images taken from the video. Firstly he set up road background relative to the different serial image. Then by using background division he segmented vehicle region and calculated the moment invariant features. These features were passed as an input to the BP neural networks. The network is divided into three layers such as Light Vehicle, Heavy Vehicle and Intermediate Vehicle. The neural network helped in classifying the vehicle type more efficiently and effectively.

Lai, Huang, and Tseng [7] used a technique of three basic steps. These steps are used by any of the vehicle classification techniques. The steps were vehicle region extraction, vehicle tracking and classification. They adopted the technique of background subtraction in order to extract the foreground. By applying some geometric parameters they removed the false edges and shadows from the image. After vehicle detection, tracking method is used for creating the correspondence between the detected vehicles at different time intervals. Vehicle classification is done by using two metrics such as aspect ratio and level of compactness.

\section{CURRENT VIDEO BASED VEHICLE CLASSIFICATION ALGORITHMS}

Automatic classification of vehicles have large number of real world applications in different fields such as toll plaza, traffic surveillance, traffic congestion avoidance, terrorist activity surveillance, avoidance of car robbery etc. Numerous algorithms are available for automatic detection and classification of vehicles. Each algorithm has its own measuring criteria in order to detect different types of vehicles. Current algorithms are classifying vehicles but there accuracy rate is still far from 100 percent. In this research paper we have discussed 6 most recent vehicle classification techniques as highlighted in Fig.2. These techniques helped more effectively and efficiently in detecting the vehicles. 
The techniques are as follows:

a) Automatic Traffic Surveillance System

1. Lane Dividing Lines Detection Algorithm

2. Horizontal Line-Based Shadow-Elimination Algorithm

3. Vertical Line-Based Shadow-Elimination Algorithm

4. Classification by using Vehicle Library

b) Neural Network-Based Classification

c) Eigenface Classification Methodology

d) Hough Line Feature Classification

e) Partial Gabor Filter Bank Classification

f) Hybrid Dynamic Bayesian Network Classification

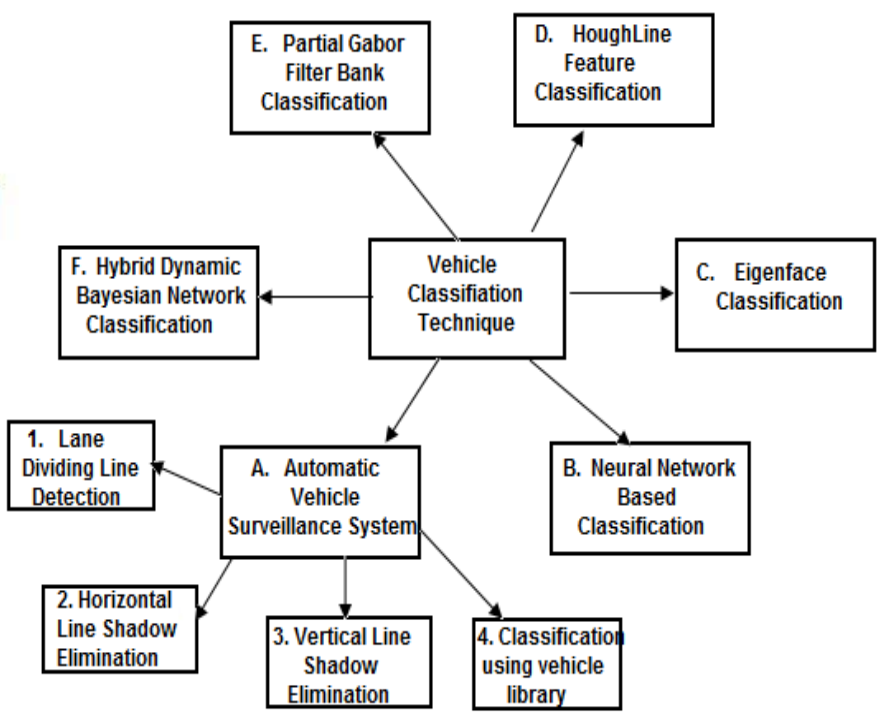

Figure. 2 Vehicle Classification Techniques

\section{A. Automatic Traffic Surveillance System}

This technique helps in classifying vehicles by using size and linearity features of vehicles [8]. It introduced new feature based on linearity. This system also designed an algorithm to remove vehicle occlusions caused by shadow. While size classification technique helps in recognizing different types of vehicles. The linearity feature helps in recognizing the difference between truck and buses though both have same size. Linearity feature extracts the un-slanted edges of the vehicles.

Major steps for this system are outlined in Fig.3. This technique firstly gets input from video in the form of frames. After that system detects various vehicles by using image differencing and background update technique then calculates vehicle histogram by accumulating the number of vehicles to certain position and detects lane dividing line. This information behaves like an input for shadow elimination algorithm. Basically horizontal and vertical line based shadow elimination algorithm is applied. The Kalman filter is applied to the output obtained from shadow elimination and it is used to track the vehicles. After that extract features of vehicles and classify vehicles based on size and linearity feature. Linearity feature is used to differentiate between truck and van truck/bus [8]. But the drawback of this proposed system is that it is affected by varying lighting conditions.

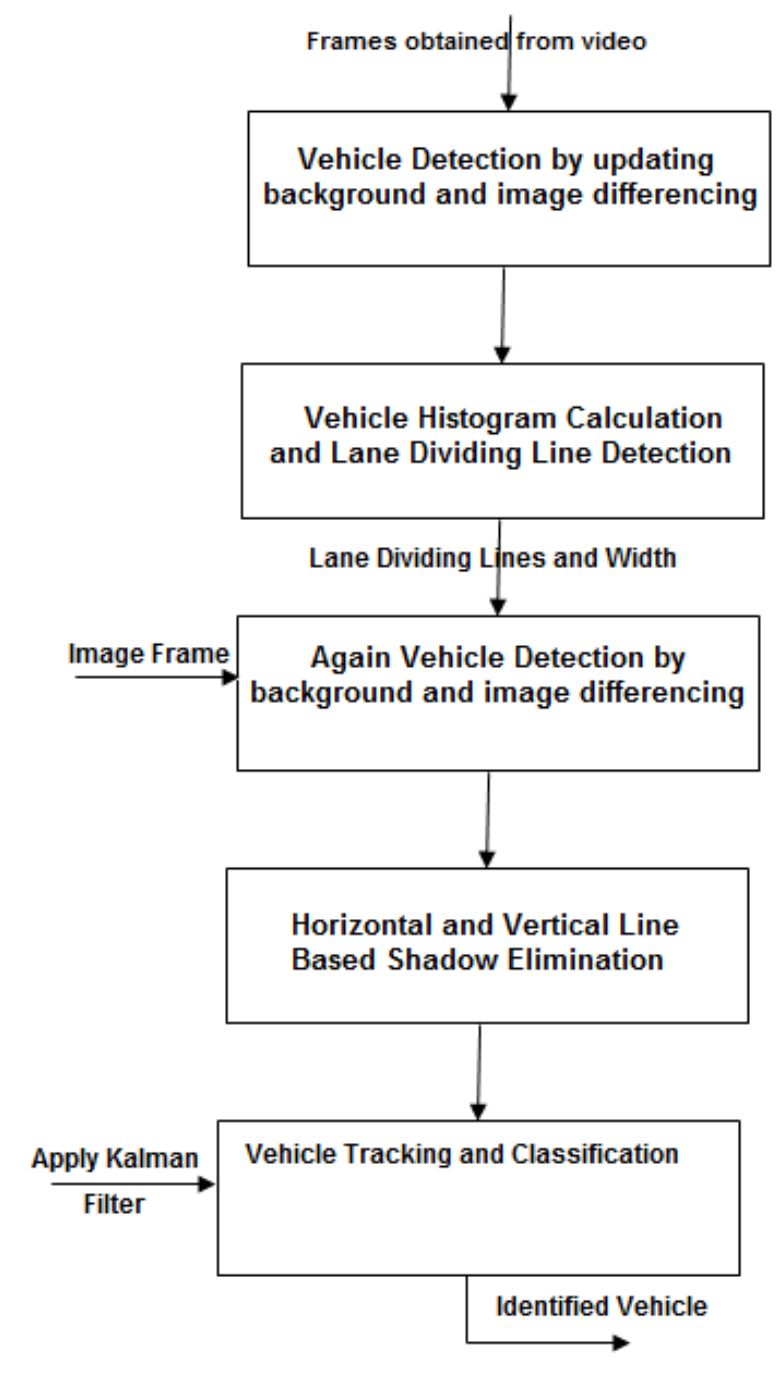

Figure. 3 Steps of Automatic Traffic Surveillance System

\section{B. Neural Network-Based Classification}

Neural network based technique is used for automatic vehicle classification. According to Fig.4, firstly this system extracts different structural features of given vehicle. These features are extracted by capturing the video of vehicles from different angles. Then normalize these images and based on these normalized features the classifier helps in classifying the given image into one of the given vehicle type. Features used for classification are structural features and the classifier is based on training. System used classifier which is based on new training method that is DSM (Direct Solution Method). The system modified the original DSM by adding more neurons to the original learning method. By making the comparison of three training methods namely Back Propagation, Direct Solution Method and Direct Solution Method with added neurons and show the classification rate in percentage for all three methods [9]. The proposed 
system classifies only three types of vehicles van, bus or car. Features used in the system are shape features and for extracting these features it uses system known as Hierarchical image process. (Shape features). This algorithm no doubt extracts vehicles but it doesn't handle shadow problem and natural weather conditions.

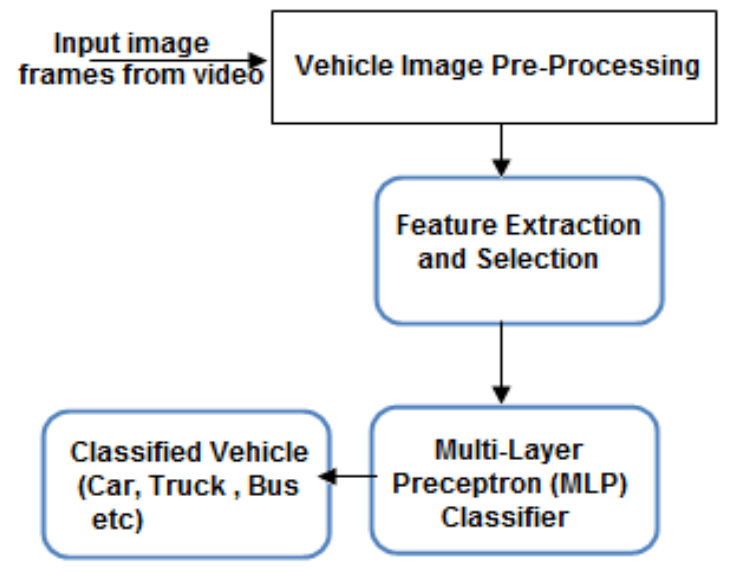

Figure. 4 Procedure of Neural Network Classification

\section{Eigenface Classification}

A technique consists of two parts: training and classification [10]. In training use the background model to get outline of vehicle and based on this outline determine right, left and bottom border of vehicle, height is set to fixed length. Image library of vehicle face is built. Eigenface technique is used to built vehicle feature library, later on this library is used in classification step i.e. features of given vehicles are extracted using eigenvector method and then based on difference between stored features and current features vehicles are classified. Results are affected by real world conditions such as rain and fog etc. (face features (left right bottom border size). The steps involve in Eigenface classification technique are shown in Fig.5.

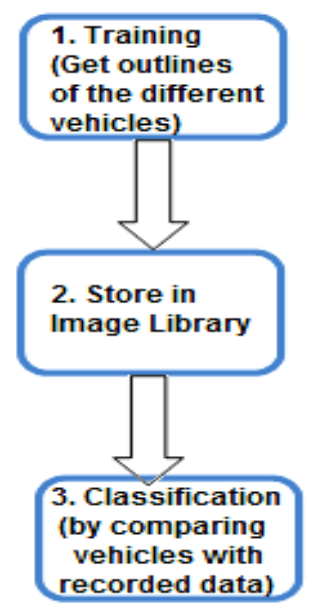

Figure. 5 Procedure of Eigenface Classification Technique

\section{Houghline Feature Classification}

This classification technique is related to recognizing people and vehicles [11]. Hough line transformation is used to detect straight lines or curves in any binary image. The steps involved in Hough line algorithm are mentioned in Fig.6. At first step capture the frames from the video then get the region of interest (ROI) in second step. In third step apply the Gaussian model for subtracting the background from the image. Edges of the object are detected by using canny operator and lines of edges can be retained from Hough line feature classification technique. It has strong anti-interference capability. But in certain cases wrong results are obtained when part of moving object enters into region of interest then it makes unstable line character.

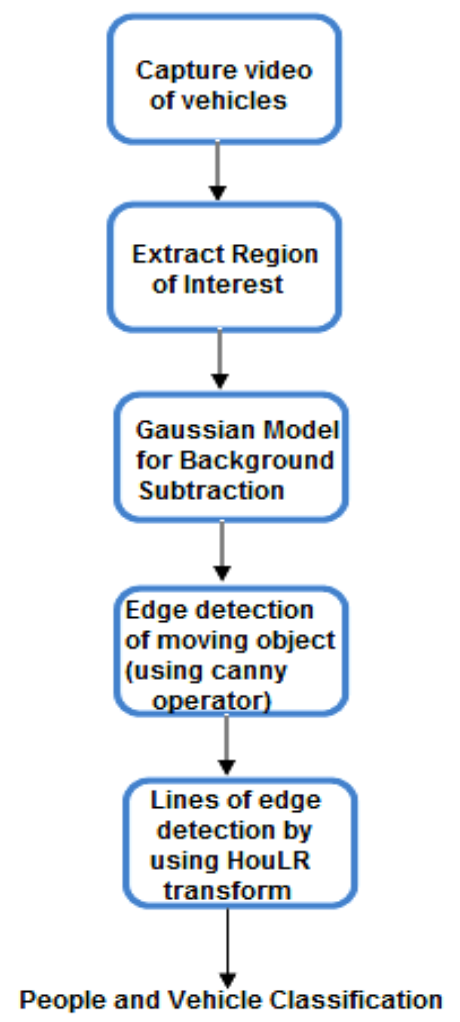

Figure. 6 Hough Line Feature Classification Steps

\section{E. Partial Gabor Filter-Based Classification}

More and more researchers divert their attention to vision-based classification in order to overcome the problems of hardware based vehicle classification. Utilize different digital cameras detector for supporting vision based classification. Generally, there are three phases in a vision-based vehicle classification system: 1) vehicle segmentation 2) feature extraction and 3) vehicle classification. Gabor filter have been generally applied in the area of pattern recognition due to its optimal localization capability in both spatial analysis and frequency domain. Due to high memory requirement and computational burden Gabor filter becomes inefficient so partial Gabor filter is used. As there is a difference among vehicles such as shape, size etc. Some vehicles are quite identical and the ratio of noise is greater at the upper side of vehicle. This cause difficulty in vehicle recognition so to avoid it instead of sample the whole image just sample the $68.0 \%$ of the top side of the image. 
Sedan, van, hatchback sedan, bus and van truck are easily classified by using this algorithm [12]. It is observed that extracting Gabor feature from edge image produces better result than grey-image of vehicles as shown in Fig.7.

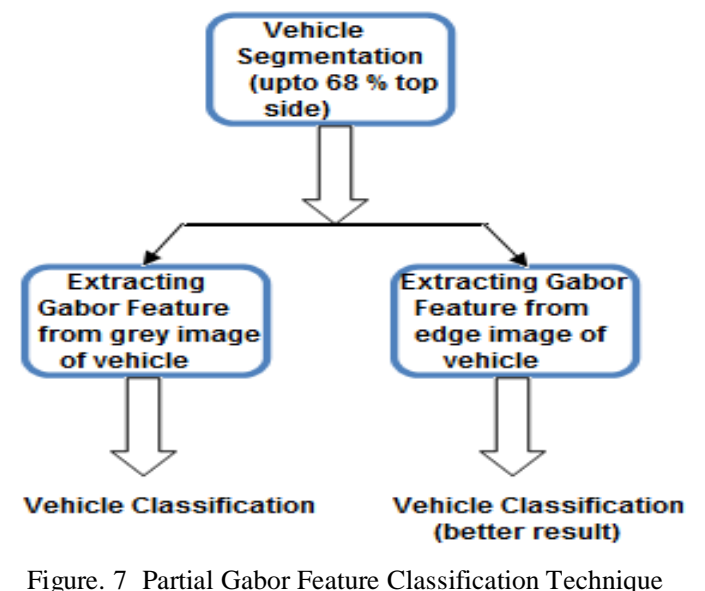

\section{F. Hybrid Dynamic Bayesian Classification (HDBC)}

A classification technique which uses direct rear view of the vehicles because rear view is less investigated and not all the countries require a front license plate [13]. Fig. 8 involves four basic stages of classification technique. Initially capturing the videos of different types of vehicles such as pick-ups, sedan, SUV's and some other vehicles categorized as unknown vehicles. Video of vehicles is captured from rear view. After capturing, extract the features of different vehicles based on rear view. In third phase select the desired feature and classify the vehicles. The results are shown in the database of 169 videos regarding these vehicles.

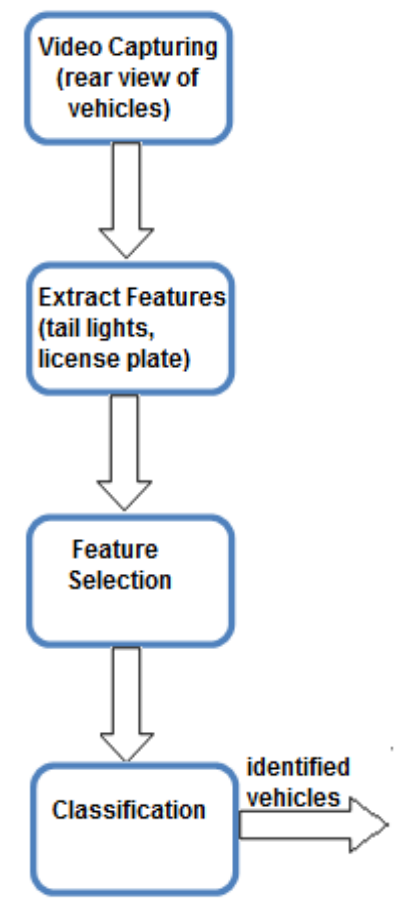

Figure. 8 Phases of Hybrid Dynamic Bayesian Network Classification Technique

\section{COMPARATIVE ANAlysis OF AUTOMATIC VEHICLE BASED CLASSIFICATION ALGORITHMS}

In this section we analyze the previously described automatic video based vehicle classification algorithms. Table II indicates the relative comparison of these techniques on the basis of different metrics. By comparing and analyzing these algorithms we concluded that Hybrid Dynamic Bayesian Classification (HDBC) is far better than the other algorithms. As HDBC have greater correction rate by extracting rear view data of vehicle.

Fig. 8 shows the three important phases of HDBC algorithm [13] such as:
a) Feature Extraction
b) Feature Selection
c) Classification

\section{a) Feature Extraction}

In feature extraction, three main types of features are extracted from the video frame images. Firstly extraction of vehicle edges by applying Gaussian mixture model along with AND mixture model. Erroneous details such as shadows are also removed from the image. The coordinates of license plate are used as an input for HDBC algorithm. In order to detect the license plate more effectively a blob detection mechanism is used along with filtering method. At the end tail lights detection technique is used for the extraction of different types of vehicles. As the distance between tail lights help in recognizing the image more efficiently.

\section{b) Feature Selection}

Feature Selection is performed in order to improve the accuracy of vehicle classification. In this case Sequential Floating Forward Selection (SFFS) method is used. In this method the most significant feature has been added in queue while removes the least significant information.

\section{c) Classification}

Dynamic Bayesian network (DBN) is used for classification of vehicles. Bayesian network uses nodes and edges; root node indicates the vehicle while other nodes describe the extracted features of the vehicles. In DBN, time dimension is involved in order to extract the information of same vehicle at different time interval and that helps in better classification of vehicles.

Each algorithm followed special methodology or technique on the basis of which recognized the vehicles. The recognition rate or the percentage accuracy of techniques are different depending upon the methodology being adopted by algorithms. Classifiers are basically the categories or types of vehicles that are identified by these algorithms. Each technique contains different vehicle samples in order to obtain the overall accuracy rate of the vehicles. The complete detail is extracted and outlined in TableII. 
TABLE II . COMPARATIVE ANALYSIS OF VEHICLE CLASSIFICATION ALGORITHMS

\begin{tabular}{|c|c|c|c|c|c|}
\hline $\begin{array}{c}\text { Technique/ } \\
\text { Classification Name }\end{array}$ & $\begin{array}{l}\text { Methodology and } \\
\text { Principles }\end{array}$ & $\begin{array}{l}\text { Accuracy } \\
\text { \%age of Vehicle } \\
\text { Classification }\end{array}$ & Classifiers & $\begin{array}{l}\text { Total Vehicle } \\
\text { Samples }\end{array}$ & Identified Flaws \\
\hline \multirow{5}{*}{$\begin{array}{l}\text { Automatic Traffic } \\
\text { Surveillance System }\end{array}$} & Front view & \multirow{5}{*}{$\begin{array}{l}82 \% \text { with shadow } \\
\text { elimination but } 69 \% \\
\text { without elimination }\end{array}$} & \multirow{5}{*}{$\begin{array}{l}\text { Car, MiniVan, } \\
\text { Van truck, truck }\end{array}$} & \multirow{5}{*}{$\begin{array}{l}\text { Total vehicle count } \\
\text { is } 20443\end{array}$} & \multirow{5}{*}{$\begin{array}{l}\text { Affected by varying } \\
\text { lighting conditions }\end{array}$} \\
\hline & Lane Division & & & & \\
\hline & Shadow removal & & & & \\
\hline & Linearity & & & & \\
\hline & Size & & & & \\
\hline \multirow{2}{*}{ Neural Network } & Neural Network & \multirow{2}{*}{$\begin{array}{l}100 \% \text { on training set } \\
\text { while } 62 \% \text { test-set }\end{array}$} & \multirow{2}{*}{ 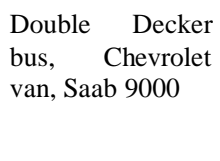 } & \multirow[t]{2}{*}{400 samples taken } & \multirow{2}{*}{$\begin{array}{l}\text { Doesn't handle shadow } \\
\text { problem and natural } \\
\text { weather conditions. }\end{array}$} \\
\hline & $\begin{array}{ll}\text { MLP } & \text { classifier } \\
\text { network } & \end{array}$ & & & & \\
\hline \multirow[t]{2}{*}{ Eigenface } & $\begin{array}{l}\begin{array}{l}\text { right, } \\
\text { bottom } \\
\text { view, }\end{array} \\
\text { border } \\
\text { biend }\end{array}$ & \multirow{2}{*}{$\begin{array}{l}100 \% \text { for training set } \\
\text { but not implemented } \\
\text { in test set }\end{array}$} & \multirow[t]{2}{*}{$\begin{array}{l}100 \text { vehicle face } \\
\text { images }\end{array}$} & \multirow[t]{2}{*}{$\begin{array}{l}100 \text { vehicles based } \\
\text { face library }\end{array}$} & \multirow{2}{*}{$\begin{array}{l}\text { Not handles real world } \\
\text { conditions such as rain } \\
\text { and fog etc }\end{array}$} \\
\hline & $\begin{array}{l}\text { Eigenvector } \\
\text { method }\end{array}$ & & & & \\
\hline \multirow{4}{*}{ Hough Line Feature } & & \multirow{4}{*}{$\begin{array}{l}97 \% \text { (in images) } \\
\text { while in video set it is } \\
\text { varying between } 91 \% \\
\text { to } 94 \% \text { (from specific } \\
\text { locations) }\end{array}$} & \multirow{4}{*}{$\begin{array}{l}\text { Location based } \\
\text { detection of any } \\
\text { type of vehicle }\end{array}$} & \multirow[t]{4}{*}{115 objects } & \multirow{4}{*}{$\begin{array}{l}\text { wrong results are } \\
\text { obtained when part of } \\
\text { moving object enters } \\
\text { into region of interest }\end{array}$} \\
\hline & $\begin{array}{l}\text { Hough line } \\
\text { transformation }\end{array}$ & & & & \\
\hline & Gaussian Model & & & & \\
\hline & Canny operator & & & & \\
\hline \multirow[t]{2}{*}{$\begin{array}{l}\text { Partial Gabor Filter } \\
\text { Based }\end{array}$} & $\begin{array}{l}\text { Grey image, edge } \\
\text { image }\end{array}$ & \multirow{2}{*}{$\begin{array}{l}95.17 \% \text { (for edge } \\
\text { images of vehicles) } \\
\text { while } 89.57 \% \text { (for } \\
\text { vehicle grey-images) }\end{array}$} & \multirow{2}{*}{$\begin{array}{l}\text { Sedan, Van, } \\
\text { Hatchback sedan, } \\
\text { Bus, Van truck }\end{array}$} & \multirow[t]{2}{*}{ Total 1196 samples } & \multirow[t]{2}{*}{$\begin{array}{l}\text { No occlusion ,shadow, } \\
\text { rain problems handled }\end{array}$} \\
\hline & $\begin{array}{l}68 \% \text { upper image } \\
\text { detail }\end{array}$ & & & & \\
\hline \multirow[t]{2}{*}{$\mathrm{HDBC}$} & Rear view & \multirow{2}{*}{$\begin{array}{l}97.63 \% \text { for Correct } \\
\text { Classification Rate } \\
\text { With approx } 2 \% \text { of } \\
\text { False Alarm Rate }\end{array}$} & \multirow{2}{*}{$\begin{array}{l}\text { Different types of } \\
\text { Sedan, Pick-up, } \\
\text { SUV/minivan and } \\
\text { unknown vehicle } \\
\text { (for other } \\
\text { vehicles) }\end{array}$} & \multirow{2}{*}{$\begin{array}{l}845 \text { images }(169 \\
\text { real-time vehicle } \\
\text { videos and } 5 \text { frames } \\
\text { each, } 169 * 5=845 \\
\text { images) }\end{array}$} & \multirow{2}{*}{$\begin{array}{l}\text { No major flaw. } \\
\text { Overcome the problem } \\
\text { of K2 algorithm. } \\
\text { Camera position can } \\
\text { cause problem }\end{array}$} \\
\hline & $\begin{array}{l}\text { Hybrid Dynamic } \\
\text { Bayesian Network }\end{array}$ & & & & \\
\hline
\end{tabular}

The percentage accuracy of video based vehicle classification algorithms are represented in the form of graph in Fig. 9. In graph, each algorithm contains two series. Automatic surveillance system based algorithms is tested under both with and without shadow elimination circumstances. But its output is affected by varying lightning conditions that's why shadow elimination produced better result. Neural Network and Eigenface algorithms have shown remarkable results under training set but these are not suitable under test set. The results of HoughLine and Partial Gabor Filter algorithm were almost same. By identifying the different features and flaws we concluded that HDBC has lower false rate i.e. approx. $2 \%$ [13]. And it is far better than the others due to its nature of estimating the minor details and classifies the variety of vehicles from video.

The overall accuracy percentage of HDBC algorithm is $97.63 \%$ and this accuracy was estimated by using 845 sample images (Total real time vehicle videos were 169 and 5 frames of each vehicle. Total images taken for the vehicles $=169 * 5=845$ images $)$. 


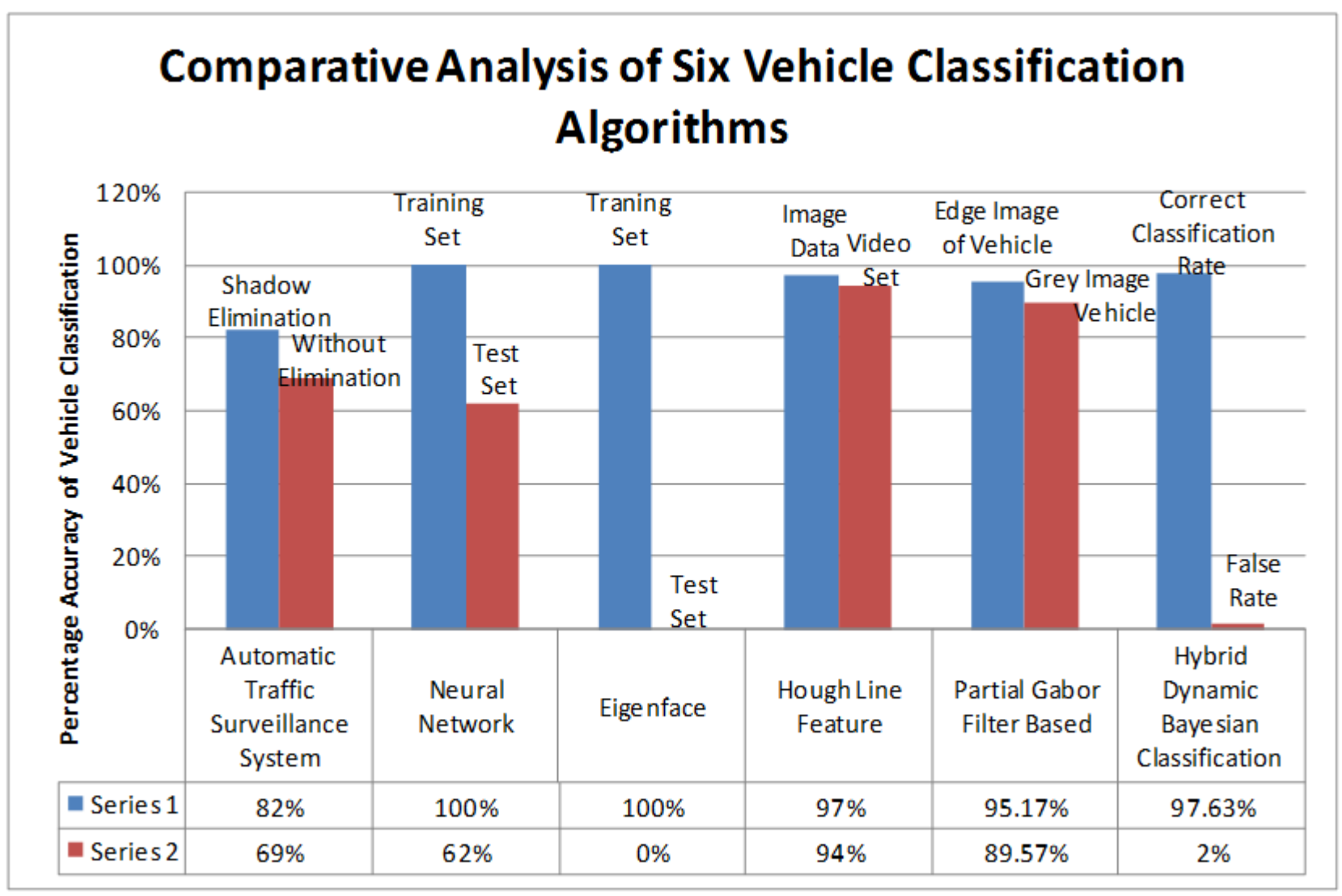

Figure. 9 Graph for Comparative Analysis of Six important Vehicle Classification Algorithms

\section{CONCLUSIONS}

Compared to the hardware based vehicle classifications software (video) based vehicle classification helps in resolving lots of issues in transportation system. This research paper is related to the comparative analysis of six well-known video based vehicle classification algorithms. On the basis of various factors (such as methodology used for these algorithms, vehicle detection rate, number of samples taken for identifying the vehicles and flaws identification in these algorithms) we have concluded that Hybrid Dynamic Bayesian Network Classification (HDBN) algorithm has better detection rate than remaining five algorithms. It has $2 \%$ of false rate which is quite lesser than other compared algorithms. It proves that high level features are not always required for gaining the greater accuracy rate. We can use simple features such as rear view of vehicle by identifying license plate and distance between the tail lights for identifying the vehicles more efficiently and effectively than any other vehicle classification algorithm.

\section{ACKNOWLEDGMENT}

We cordially thank our honorable supervisor Engr. Ali Javed for guiding us with his useful suggestions and experience. These suggestions helped us in the completion of this research paper.

\section{REFERENCES}

[1] Kostia Robert, "Night-Time Traffic Surveillance A Robust Framework for Multi-Vehicle Detection, Classification and Tracking", Advanced Video and Signal Based Surveillance, IEEE, 2009

[2] Ryan P. Avery, Yinhai Wang, and G. Scott Rutherford," Length-Based Vehicle Classification Using Images from Un-calibrated Video Cameras", Intelligent Transportation Systems, 2004 Proceedings. The 7th International IEEE Conference

[3] Guohui Zhang, Ryan P. Avery, Yinhai Wang, "A Video-based Vehicle Detection and Classification System for Real-time Traffic Data Collection Using Uncalibrated Video Cameras", National Research Council, Washington, DC , 2007

[4] Kun Wu, Haiying Zhang, Tianmao Xu, Ju Song, "Overview of Video-Based Vehicle Detection Technologies", The 6th International Conference on Computer Science \& Education (ICCSE 2011) August 3-5, 2011. SuperStar Virgo, Singapore

[5] Yang Wang,, "Real-Time Moving Vehicle Detection With Cast Shadow Removal in Video Based on Conditional Random Field", IEEE transactions on circuits and systems for video technology, vol. 19, no. 3, March 2009

[6] Zhong Qin," Method of Vehicle Classification Based on Video", Proceedings of the 2008 IEEE/ASME 
International Conference on Advanced Intelligent Mechatronics July 2 - 5, 2008, Xi'an, China

[7] Jin-Cyuan Lai, Shih-Shinh Huang, and Chien-Cheng Tseng , "Image-Based Vehicle Tracking and Classification on the Highway" Green Circuits and Systems (ICGCS), 2010 International Conference, 21-23 June 2010

[8] Jun-Wei Hsieh, Shih-Hao Yu, Yung-Sheng Chen, Wen-Fong $\mathrm{Hu}$ "Automatic Traffic Surveillance System for Vehicle Tracking and Classification", IEEE Transactions On Intelligent Transportation Systems, Vol. 7, No. 2, June 2006

[9] Anshul Goyal and Brijesh Verma ,“ A Neural Network based Approach for the Vehicle Classification", Proceedings of the 2007 IEEE Symposium on Computational Intelligence in Image and Signal Processing (CIISP 2007)

[10] Wei Wang ,Yulong Shang ,Jinzhi Guo,Zhiwei Qian” Real-time Vehicle Classification Based on Eigenface", 2011 IEEE

[11] Tao Xu, Hong Liu, Yueliang Qian and Han Zhang, "A Novel Method for People and Vehicle Classification Based on Hough Line Feature", International Conference on Information Science and Technology March 26-28, 2011 Nanjing, Jiangsu, China

[12] Peijin Ji, Lianwen Jin, Xutao Li," Vision-based Vehicle Type Classification Using Partial Gabor Filter Bank", Proceedings of the IEEE International Conference on Automation and Logistics August 18 - 21, 2007, Jinan, China

[13] Mehran Kafai , Bir Bhanu "Dynamic Bayesian Networks for Vehicle Classification in Video", IEEE Transactions On Industrial Informatics, Vol. 8, No. 1, February 2012.

\section{AUTHOR'S PROFILE}

Engr Kanwal Yousaf is MSc Scholar in Department of Software Engineering at University of Engineering and Technology, Taxila. She completed her Bachelor's degree in Software Engineering from UET, Taxila in 2010. She is currently working on Web 2.0 based Elearning by using social media. Her area of interest is Software Quality Assurance, Internet application development, Digital Image Processing and Wireless networks.

Engr. Arta Iftikhar is MSc Scholar in Departement of Software Engineering at University of Engineering and Technology Taxila. She completed her Bachelor's degree in Software Engineering from University of Engineering and Technology Taxila in 2011. She is currently working on "Efficient Algorithm for Vehicle Classification". Her area of interest is Digital Image Processing, Database and Wireless Networks.
Engr. Ali Javed has been an Assistant Professor since March 2012 in the Department of Software Engineering, University of Engineering and Technology Taxila, Pakistan. He accomplished his M.Sc in Computer engineering from university of Engineering and Technology Taxila, Pakistan in February, 2010. His areas of interest are Video Summarization, Digital Image Processing, Computer vision, Software Quality Assurance, Software testing and Software Requirements Analysis. 